Zhi Feng Zhang*, Rui Wang, Fei Ye, Haibin Wang and Wenxia Zhao

\title{
Extraction of glycyrrhizic acid by aqueous two-phase system formed by PEG and two environmentally friendly organic acid salts - sodium citrate and sodium tartrate
}

https://doi.org/10.1515/gps-2019-0024

Received November 8, 2018; accepted March 27, 2019.

\begin{abstract}
Two aqueous two-phase systems (ATPS's) formed by using PEG and sodium citrate/sodium tartrate are applied and compared for extraction of glycyrrhizic acid (GA) from its stock solution. Their binodal curves and tie-lines are studied firstly. Influence of usage amount of the salt and the PEG on the partition coefficient and extraction efficiency is investigated. The highest extraction efficiency and partition coefficient achieved is $73 \%$ and 6.5, when the sodium citrate and PEG concentration is $15 \%$ and $20 \%$ respectively. It is also found that the ATPS based on sodium citrate is better than sodium tartrate for GA extraction. The present study indicates that ATPS formed by biodegradable organic acid salts could be feasible and environment-friendly technique for GA and other bioactive compounds.
\end{abstract}

Keywords: glycyrrhizic acid; licorice; aqueous two-phase system; extraction

\section{Introduction}

Licorice, the root of Glycyrrhiza glabra, is one of the most important herbs in the traditional Chinese medicine (TCM) and appears in a large number of TCM prescriptions. Glycyrrhizic acid (GA) is one of the predominant bioactive components in licorice. In the past few decades, bioeffects of GA have been extensively studied and several beneficial effects are confirmed. It has been found that GA is anti-virus [1], anti-inflammatory [2,3], anti-allergic [4],

* Corresponding author: Zhi Feng Zhang, College of Chemistry and Chemical Engineering, Ningxia Normal University, Guyuan, Ningxia, China, 756000, e-mail: 82016001@nxnu.edu.cn

Rui Wang, Fei Ye, Haibin Wang and Wenxia Zhao, College of Chemistry and Chemical Engineering, Ningxia Normal University, Guyuan, Ningxia, China, 756000 anti-cancer [5] etc. In addition, recent studies reveal that GA also has markedly hepatoprotective effects [6,7]. Thus, facile and environment-friendly extraction of GA from licorice is practically needed.

Among various extraction and separation techniques, Soxhlet extraction of GA at ambient pressure is still the most widely used method due to its simplicity. Normally, water is used as the extraction solvent. To improve extraction ratio, selectivity and recovery, other solvents like methanol or ethanol are added [8]. To further enhance extraction, microwave-assisted extraction [9], microwaveassisted micellar extraction [10], ultrasound-assisted extraction [11,12], cloud point extraction [13], and multistage countercurrent extraction [14] etc are investigated and reported in literature. Another major extraction technique is supercritical fluid extraction. It has been used for separation because supercritical fluid has high dissolving power and low viscosity. Supercritical $\mathrm{CO}_{2}$ has been applied to extract GA and an extraction recovery of $54 \%$ is achieved. Superheated water is also utilized for GA extraction and it is found that the amount of extracted GA by superheated water is higher than Soxhlet method. Nevertheless, the process of supercritical fluid extraction is onerous and high-cost and thus its wide application is limited.

Liquid-liquid extraction based on aqueous two-phase system (ATPS) has quite a few advantages and hence is applied for extraction of active compounds from medical plants as well as bio-macromolecules including protein and DNA/RNA [15-19]. This extraction technology is facile and low-cost, and the used chemicals are not toxic. ATPS formed by EtOH- $\mathrm{K}_{2} \mathrm{HPO}_{4}-\mathrm{H}_{2} \mathrm{O}$ [20], PEG- $\left(\mathrm{NH}_{4}\right)_{2} \mathrm{SO}_{4}-$ $\mathrm{H}_{2} \mathrm{O}$ and PEG- $\mathrm{K}_{2} \mathrm{HPO}_{4}-\mathrm{H}_{2} \mathrm{O}$ [21] and nonionic surfactant with $\mathrm{NaCl} / \mathrm{Na}_{2} \mathrm{SO}_{4} / \mathrm{Na}_{3} \mathrm{PO}_{4}$ [22] has been used to extract $\mathrm{GA}$, demonstrating excellent extraction performance. Recently, biodegradable organic salts are explored to form ATPS's to make this technology more environmentfriendly, including citrate, tartrate, formate and succinate etc [23-26]. There have been a large number of reports concerning ATPS's formed by those organic salts with PEG 
and their according phase diagrams. Taking advantage of these clean ATPS's, this paper utilizes ATPS formed by PEG-sodium citrate and PEG-sodium tartrate to extract GA from its stock solution, to investigate the feasibility of extracting GA by using these two ATPS's. These two ATPS's are selected because they are found having quite large heterogeneous region and thus good separation capability [27,28].

\section{Materials and methods}

\subsection{Materials}

PEG with molecular weight of $10000 \mathrm{~g} / \mathrm{mol}$, sodium citrate dihydrate (ACS, $\geq 99 \%$ ), and glycyrrhizic acid $(\geq 98 \%)$ are purchased from Sinopharm Chemical Reagent and used without further purification. Deionized water with an electrical conductivity of $5 \mu \mathrm{S} / \mathrm{cm}$ was purified from tap water and used. Licorice (cultivated in Guyuan, Ningxia, China) in slices was purchased from a local TCM store.

\subsection{Determination of binodal curves and tie-lines}

Binodal curves of the PEG $10000+$ sodium citrate $+\mathrm{H}_{2} \mathrm{O}$ and PEG $10000+$ sodium tartrate $+\mathrm{H}_{2} \mathrm{O}$ were measured by using the cloud point method. Ten water solutions of PEG ranging from $2 \mathrm{wt} \%$ to $45 \mathrm{wt} \%$, and a sodium citrate or sodium tartrate solution of $35 \mathrm{wt} \%$ were prepared in assay tubes and kept in a water bath maintained at $25 \pm 0.1^{\circ} \mathrm{C}$ (SYC-15B, Nanjing Sangli Electronic Instrument Co). Mass of all assay tubes with solutions was precisely measured on a precision electronic balance (FA1004N, Shanghai Precision Scientific Instrument Co) with an uncertainty of $\pm 0.0002 \mathrm{~g}$. Assay tubes were kept in the bath for $1 \mathrm{~h}$ to reach temperature equilibrium. Then salt solution was dipped into each PEG solutions until the PEG solutions became cloudy. Tubes with PEG solutions were all weighed again to determine the mass of added salt solution, so that mass fraction of the salt and the PEG in the solution was calculated. Tie-lines were measured by adding excess salt solution to PEG solutions, vigorously shaken and kept in the water bath for $48 \mathrm{~h}$ to reach phase equilibrium. Mass fraction of the salt and the PEG was determined by measuring refractive index and electrical conductivity of the top and the bottom phases. Two correlation functions were obtained by measuring refractive index and electrical conductivity of solutions with known fraction of PEG and sodium citrate/sodium tartrate. The used Abbe refractometer (WYA-2S, Shanghai Precision Scientific Instrument Co.) has a precision of 0.0002 and the measurement was carried out at $25 \pm 0.1^{\circ} \mathrm{C}$, maintained by circulating water from the water bath. The electrical conductivity meter (DDS-II A, Shanghai Leici) has a measurement error of $2 \%$. It should be noted that all experiments were conducted under the local ambient pressure of $84 \pm 1 \mathrm{kPa}$.

\subsection{Extraction of GA}

Soxhlet extraction technique was applied for the preliminary extraction of GA from licorice for its convenience and relatively high extraction efficiency. Licorice slices were dried at $60^{\circ} \mathrm{C}$ for $8 \mathrm{~h}$ in an oven, then milled and filtered by a 60 mesh screen to obtain powder sample. An amount of $10 \mathrm{~g}$ powder sample was precisely weighed on the electronic balance, wrapped up by a clean filter paper and inserted into a Soxhlet assembly filled with $100 \mathrm{~mL}$ deionized water as extraction solvent. Each Soxhlet extraction continued for $24 \mathrm{~h}$, and the extracted solution was stocked for ATPS extraction in the following step.

ATPS extraction was conducted by dripping $2 \mathrm{~g}$ of stock solution into an ATPS with known PEG and salt mass fraction in a centrifuge tube. The tube was vigorously shaken, centrifuged at 2000 rotations/min for $30 \mathrm{~min}$ and submerged in a water bath of $25^{\circ} \mathrm{C}$ for at least $24 \mathrm{~h}$ to reach phase equilibrium. Finally, the top and the bottom phases were separated by using a syringe and weighed. GA concentration in each phase was determined by diluting solution of each phase and measuring absorbance at $252 \mathrm{~nm}$. Mass fraction of PEG and salt was determined by the aforementioned method. To study the influence of PEG fraction and also salt fraction on the performance of ATPS extraction, one of them was fixed while the other was changed to measure partition coefficient and extraction efficiency.

\subsection{Measurement of GA concentration}

Concentration of GA in the top and the bottom phases after equilibrium was determined by using a UV spectrometer, since GA absorbs UV light close to $250 \mathrm{~nm}$ in ethanol/water solution. The calibration curve was obtained by measuring absorbance of GA solution with known concentration at $252 \mathrm{~nm}$. After reaching phase 
equilibrium, a small amount of solution from the top and the bottom phases was drawn by a syringe and diluted, and its absorbance was measured to calculate GA mass fraction. The standard uncertainty for GA concentration is experimentally determined as $10^{-3} \mathrm{wt} / \mathrm{wt}$.

\subsection{Extraction parameters}

The partition coefficient $K$ is defined as the ratio of GA mass fraction of the top phase (PEG-rich) over the bottom phase (salt-rich):

$$
K=\frac{w(\text { salt })^{T P}}{w(\text { salt })^{B P}}
$$

Where $w(\text { salt })^{T P}$ and $w(\text { salt })^{B P}$ is the mass fraction of GA in the top and the bottom phase, respectively.

The extraction efficiency $(E E)$ is defined as the mass of GA in the PEG-rich phase over the total mass in both phases:

$$
E E=\frac{m(G A)^{T P}}{m(G A)^{T P}+m(G A)^{B P}} \times 100 \%
$$

Where $m(G A)^{T P}$ and $m(G A)^{B P}$ is the mass of GA in the top phase and the bottom phase, respectively.

\section{Results and discussion}

\subsection{Phase diagram}

Binodal curves and tie-lines of PEG 10000 + sodium citrate $+\mathrm{H}_{2} \mathrm{O}$ and $\mathrm{PEG} 10000+$ sodium tartrate $+\mathrm{H}_{2} \mathrm{O}$ are shown in Figures 1 and 2, and the according data of tie-lines as well as tie-line length (TLL) and slope of tie-line (STL) are listed in Tables 1 and 2, respectively. For comparison, binodal curves of PEG $8000+$ sodium citrate $+\mathrm{H}_{2} \mathrm{O}$ reported in Ref and PEG $4000+$ sodium tartrate $+\mathrm{H}_{2} \mathrm{O}$ reported in Ref are plotted in two figures for comparison [25,29]. For both systems, PEG of higher molecular weight produces larger heterogeneous region. This is already confirmed by many previous studies $[27,28,30]$. It is evident that ATPS formed by sodium tartrate has larger STL than sodium citrate. To further check validity of the data, binodal curves are fitted by using the empirical equation proposed by Merchuk et al. [31]:

$$
y=a \cdot e^{b \cdot x^{0.5}-c \cdot x^{3}}
$$

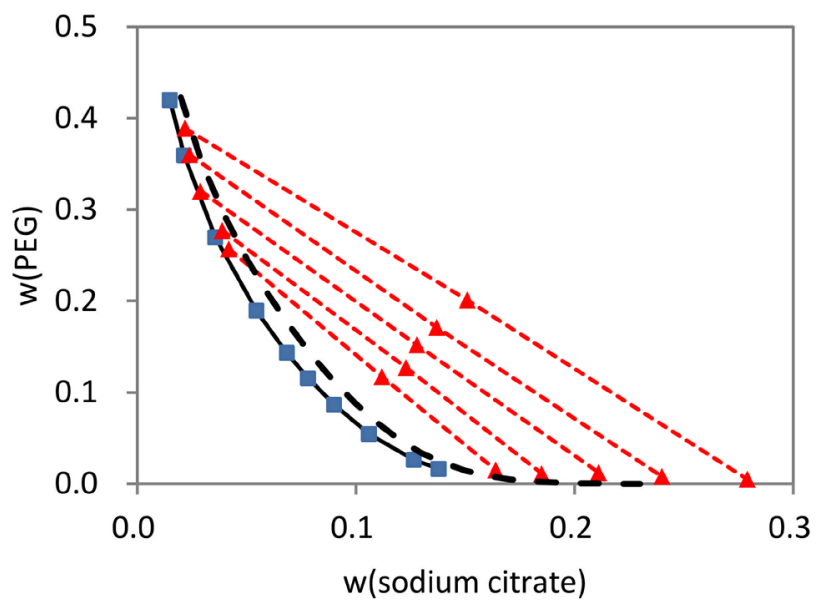

Figure 1: Binodal curve $(\square)$ and tie-line $(\boldsymbol{\Delta})$ of PEG $10000+$ sodium citrate $+\mathrm{H}_{2} \mathrm{O}$ at $\mathrm{T}=298.15 \mathrm{~K}$ and $\mathrm{P}=84 \mathrm{kPa}$. Dash line is the cloud point data in [15] (PEG $8000+$ sodium citrate $+\mathrm{H}_{2} \mathrm{O}, 298.15 \mathrm{~K}$ ) for comparison.

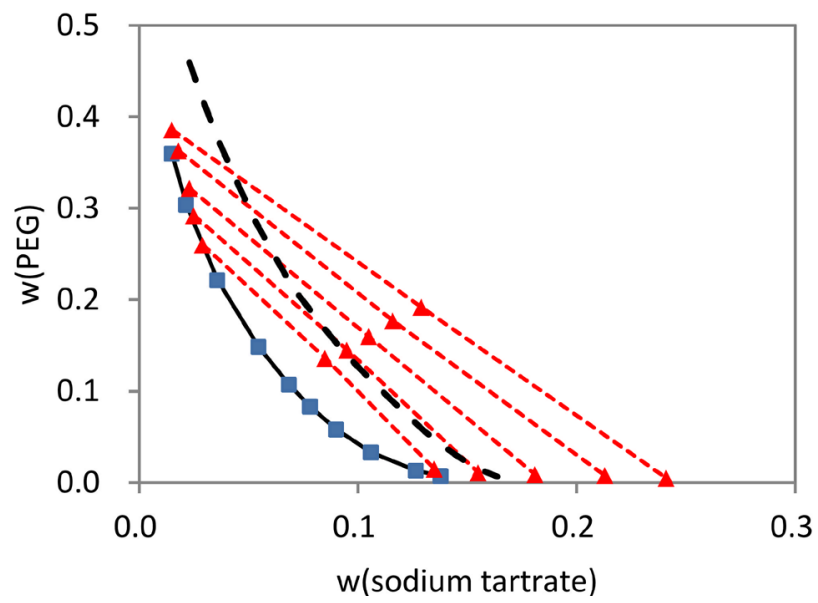

Figure 2: Binodal curve $(\boldsymbol{\square})$ and tie-line $(\boldsymbol{\Delta})$ of PEG 10000 + sodium tartrate $+\mathrm{H}_{2} \mathrm{O}$ at $\mathrm{T}=298.15 \mathrm{~K}$ and $\mathrm{P}=84 \mathrm{kPa}$. Dash line is the cloud point data in [29] (PEG $4000+$ sodium tartrate $+\mathrm{H}_{2} \mathrm{O}, 298.15 \mathrm{~K}$ ) for comparison.

Table 1: Mass fraction of feed, top and bottom phases for PEG

\begin{tabular}{|c|c|c|c|c|c|c|c|}
\hline \multicolumn{2}{|c|}{ Feed } & \multicolumn{2}{|c|}{ Top Phase } & \multicolumn{2}{|c|}{ Bottom Phase } & \multirow{2}{*}{ TLL } & \multirow{2}{*}{ STL } \\
\hline w(Salt) & $w(P E G)$ & $w($ Salt) & $w($ PEG) & $w($ Salt) & $w($ PEG) & & \\
\hline .112 & 0.117 & 0.042 & 0.257 & 0.164 & 0.015 & 0.27 & -1.98 \\
\hline 0.123 & 0.127 & 0.039 & 0.277 & 0.185 & 0.011 & 0.30 & -1.82 \\
\hline 0.128 & 0.152 & 0.029 & 0.320 & 0.211 & 0.012 & 0.36 & -1.69 \\
\hline 0.137 & 0.17 & 0.024 & 0.360 & 0.241 & 0.008 & 0.41 & -1.62 \\
\hline 0.151 & 0.201 & 0.022 & 0.389 & 0.279 & 0.005 & 0.46 & -1.49 \\
\hline
\end{tabular}
$10000+$ Sodium Citrate $+\mathrm{H}_{2} \mathrm{O}$ at $298.15 \mathrm{~K}$ and $84 \mathrm{kPa}$, and the calculated TLL and STL. ${ }^{\mathrm{a}}$

a Standard uncertainties $\mathrm{u}$ are $\mathrm{u}(\mathrm{w})=0.001, \mathrm{u}(\mathrm{T})=0.1 \mathrm{~K}, \mathrm{u}(\mathrm{P})=1 \mathrm{kPa}$. 
Where $x$ and $y$ are mass fraction of the salt and PEG respectively, and $a, b$ and $c$ are fitting parameters. The fitted values for them and the coefficient of determination $\mathrm{R}^{2}$ are presented in Table 3. The $\mathrm{R}^{2}$ for both systems are very close to unity, indicating high measurement accuracy.

\subsection{Effect of PEG concentration}

The influence of overall PEG concentration on the partition coefficient $K$ and extraction efficiency $E E$ is illustrated in Figure 3, while the slat concentration is

Table 2: Mass fraction of feed, top and bottom phases for PEG $10000+$ Sodium tartrate $+\mathrm{H}_{2} \mathrm{O}$ at $298.15 \mathrm{~K}$ and $84 \mathrm{kPa}$, and the calculated TLL and STL. ${ }^{\mathrm{a}}$

\begin{tabular}{|c|c|c|c|c|c|c|c|}
\hline \multicolumn{2}{|c|}{ Feed } & \multicolumn{2}{|c|}{ Top Phase } & \multicolumn{2}{|c|}{ Bottom Phase } & \multirow{2}{*}{ TLL } & \multirow{2}{*}{ STL } \\
\hline$w($ Salt) & $w($ PEG) & $w($ Salt) & $w(P E G)$ & $w($ Salt) & $w(P E G)$ & & \\
\hline 085 & 0.136 & 0.029 & 0.260 & & 0.015 & 0.27 & -2.31 \\
\hline .095 & 0.145 & 0.025 & 0.292 & 0.155 & 0.011 & 0.31 & -2.16 \\
\hline 105 & 0.160 & 0.023 & 0.322 & 181 & 0.009 & 0.35 & -1.98 \\
\hline 0.116 & 0.177 & 0.018 & 0.363 & 0.213 & 0.008 & 0.41 & -1.82 \\
\hline 0.129 & 0.192 & 0.015 & 0.386 & 0.241 & 0.005 & 0.44 & -1.68 \\
\hline
\end{tabular}

a Standard uncertainties $\mathrm{u}$ are $\mathrm{u}(\mathrm{w})=0.001, \mathrm{u}(\mathrm{T})=0.1 \mathrm{~K}, \mathrm{u}(\mathrm{P})=1 \mathrm{kPa}$.

Table 3: Fitted parameters of the Merchuk equation for the two studied ATPS's and coefficient of determination $\mathrm{R}^{2}$.

\begin{tabular}{lrrrr}
\hline System & $\boldsymbol{a}$ & $\boldsymbol{b}$ & $\boldsymbol{c}$ & $\mathbf{R}^{2}$ \\
\hline PEG + Sodium citrate $+\mathrm{H}_{2} \mathrm{O}$ & 0.927 & -6.25 & 657 & 0.998 \\
PEG + Sodium tartrate $+\mathrm{H}_{2} \mathrm{O}$ & 0.850 & -6.86 & 853 & 0.999 \\
\hline
\end{tabular}

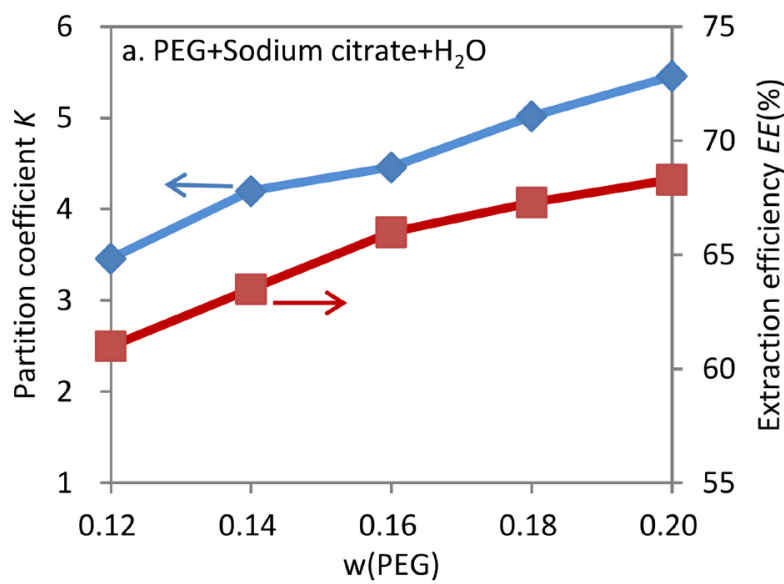

fixed at $12 \mathrm{wt} \%$. Within the studied concentration range, more PEG produces better extraction, that is, higher $K$ and $E E$. However, the increase of $E E$ becomes small as the fraction of PEG approaches $20 \%$. The reason is that the mass of PEG phase reduces compared to the bottom phase although the fraction of GA in the PEG phase rises. Practically, raising PEG concentration is limited by its solubility in water, especially for PEG with molecular weight higher than 10000. Besides, the PEG phase would become too viscous and phase separation will be too slow if PEG concentration exceeds suitable value. Considering all results, it is evident that the ATPS formed by sodium citrate is better than sodium tartrate for extracting GA.

\subsection{Effect of salt concentration}

Figure 4 shows influence of salt concentration on the partition coefficient $K$ and extraction efficiency $E E$, while the fraction of PEG is maintained at $20 \%$. $K$ ranges from 4 to 7 and increases as mass fraction of salt is raised. High salt concentration also leads to high extraction efficiency below $20 \%$. No further increase is observed for sodium citrate more than $14 \%$, while $E E$ decreases slightly for sodium tartrate based ATPS. The highest partition coefficient occurs when the mass fraction of sodium citrate and PEG are $15 \%$ and $20 \%$, respectively. In terms of $K$ and $E E$, PEG-sodium citrate is better than PEG-sodium tartrate for extraction of GA from its stock solution. It has to admit that the highest extraction efficiency achieved by the present study is lower than that by other in previous studies. For example, the highest EE of GA by other ATPSs is well above $90 \%$, such as EtOH- $\mathrm{K}_{2} \mathrm{HPO}_{4}-\mathrm{H}_{2} \mathrm{O}$ [20], PEG$\left(\mathrm{NH}_{4}\right)_{2} \mathrm{SO}_{4}-\mathrm{H}_{2} \mathrm{O}$ and PEG-K $\mathrm{HPO}_{4}-\mathrm{H}_{2} \mathrm{O}$ [21]. Nevertheless, the

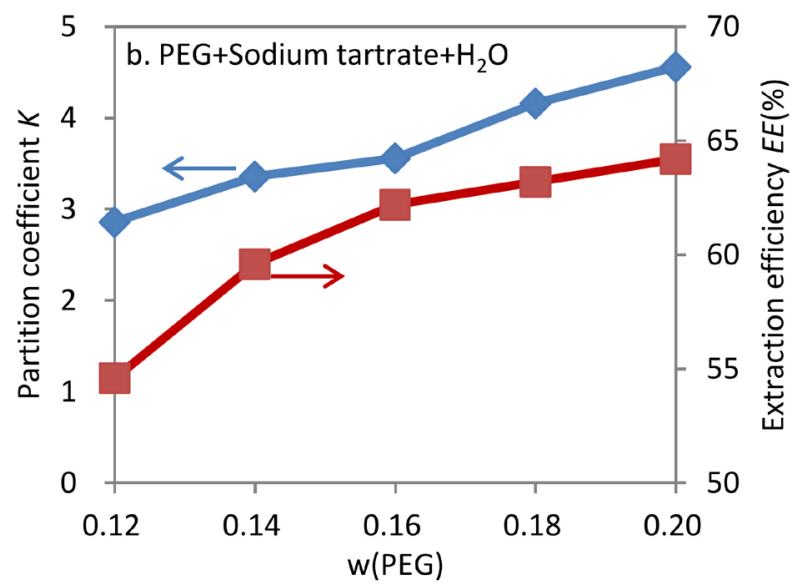

Figure 3: Dependence of Partition coefficient $K$ and extraction efficiency EE on total mass fraction of PEG in ATPS of (a) PEG $10000+$ Sodium citrate $+\mathrm{H}_{2} \mathrm{O}$ and (b) PEG $10000+$ sodium tartrate $+\mathrm{H}_{2} \mathrm{O}$. The mass fraction of sodium citrate and sodium tartrate are $12 \%$. 

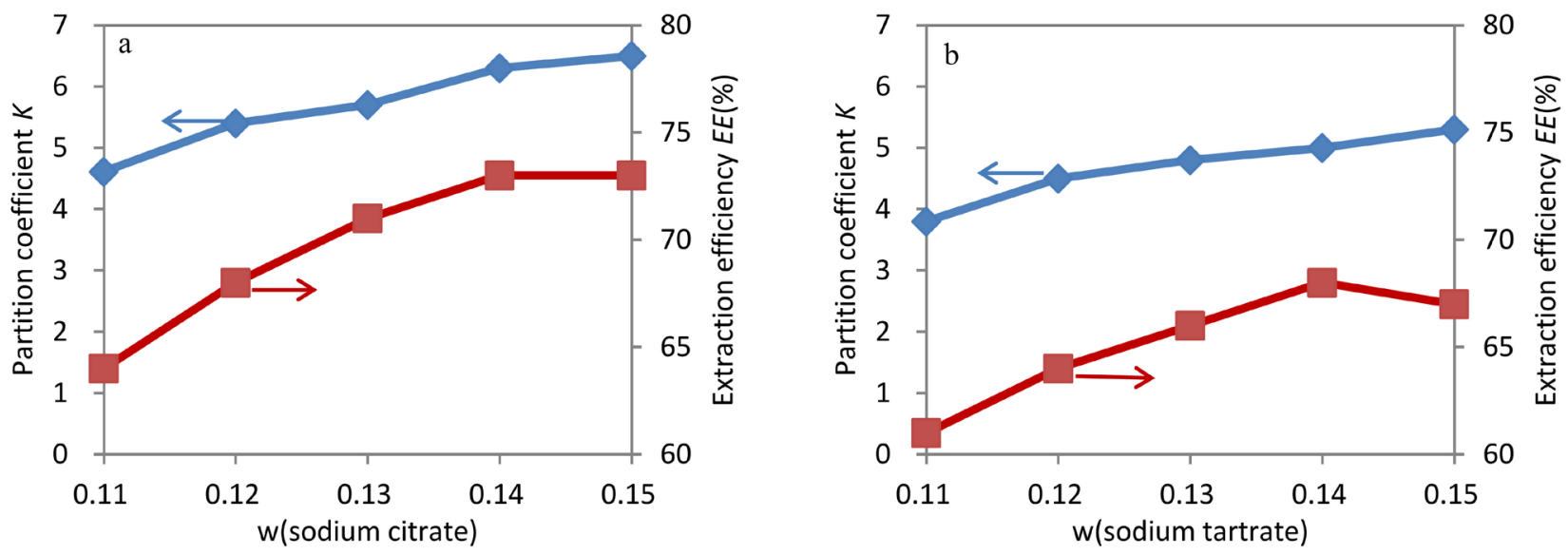

Figure 4: Dependence of Partition coefficient $K$ and extraction efficiency EE on total mass fraction of salt in ATPS of (a) PEG $10000+$ Sodium citrate $+\mathrm{H}_{2} \mathrm{O}$ and (b) PEG $10000+$ sodium tartrate $+\mathrm{H}_{2} \mathrm{O}$. The mass fraction of PEG is $20 \%$ for the both systems.

EE of GA for PEG + sodium citrate $+\mathrm{H}_{2} \mathrm{O}$ can be improved by further optimizing the extraction conditions like salt and PEG concentration, temperature, $\mathrm{pH}$, etc.

\section{Conclusions}

Extraction of GA from its stock solution by using ATPS formed by PEG 10000 and two organic acid salts, namely, sodium citrate and sodium tartrate, are studied. Binodal curves and tie-lines of two ATPS's are experimentally measured. The influence of concentration of PEG and salts on two extraction parameters is investigated, including partition coefficient and extraction efficiency. Results indicate that both ATPS's are effective in extraction of GA from the stock solution, while the ATPS of sodium citrate is slightly better than sodium tartrate. Among all experiments, the highest extraction efficiency is $73 \%$ by PEG 10000 + sodium citrate $+\mathrm{H}_{2} \mathrm{O}$ while the mass fraction of sodium citrate and PEG are $15 \mathrm{wt} \%$ and $20 \mathrm{wt} \%$, respectively. The overall extraction efficiency could be dramatically improved by using ultrasound-assisted or microwave-assisted extraction since the present study just used the most basic Soxhlet extraction. Nevertheless, this study shows ATPS formed by bio-degradable organic acid salts could be a feasible and environment-friendly technique for extraction of GA from licorice.

Acknowledgement: Zhi Feng Zhang acknowledges the financial support of this research by funding from Ningxia Normal University under grant No. NXSFZDA1808, and its College of Chemistry and Chemical Engineering under grant No. HGZD18-03. Haibin Wang acknowledges the financial support by Ningxia Education Department under grant No. NGY2016197 and National Natural Science Foundation of China under grant No. 31860518. Wenxia Zhao acknowledges the financial support by National Natural Science Foundation of China under grant No. 21561027.

The authors declare no competing financial interest.

\section{Reference}

[1] Pompei R., Flore O., Marccialis M.A., Pani A., Loddo B., Glycyrrhizic acid inhibits virus growth and inactivates virus particles. Nature, 1979, 281, 689-690.

[2] Bhattacharjee S., Bhattacharjee A., Majumder S., Majumdar S.B., Majumdar S., Glycyrrhizic acid suppresses Cox-2mediated anti-inflammatory responses during Leishmania donovani infection. J. Antimicrob. Chemother., 2012, 67, 1905-1914.

[3] Li Y., Sun F., Jing Z., Wang X., Hua X., Wan L., Glycyrrhizic acid exerts anti-inflammatory effect to improve cerebral vasospasm secondary to subarachnoid hemorrhage in a rat model. Neurol. Res., 2017, 39, 727-732.

[4] Han S., Sun L., He F., Che H., Anti-allergic activity of glycyrrhizic acid on IgE-mediated allergic reaction by regulation of allergyrelated immune cells. Sci. Rep., 2017, 7, 7222.

[5] Chueh F.S., Hsiao Y.T., Chang S.J., Wu P.P., Yang J.S., Lin J.J., et al., Glycyrrhizic acid induces apoptosis in WEHI-3 mouse leukemia cells through the caspase- and mitochondria-dependent pathways. Oncol. Rep., 2012, 28, 2069-2076.

[6] Orazizadeh M., Fakhredini F., Mansouri E., Khorsandi L., Effect of glycyrrhizic acid on titanium dioxide nanoparticlesinduced hepatotoxicity in rats. Chem. Biol. Interact., 2014, 220, 214-221. 
[7] Sun X., Duan X., Wang C., Liu Z., Sun P., Huo X., et al., Protective effects of glycyrrhizic acid against non-alcoholic fatty liver disease in mice. Eur. J. Pharmacol., 2017, 806, 75-82.

[8] Tian M., Yan H., Row H.K., Extraction of glycyrrhizic acid and glabridin from Licorice. Int. J. Mol. Sci., 2008, 9, 571-577.

[9] Pan X., Liu H., Jia G., Shu Y.Y., Microwave-assisted extraction of glycyrrhizic acid from licorice root. Biochem. Eng. J., 2000, 5, 173-177.

[10] Sun, C., Xie Y., Liu H., Microwave-assisted micellar extraction and determination of glycyrrhizic acid and liquiritin in licorice root by HPLC. Chin. J. Chem. Eng., 2007, 15, 474-477.

[11] Charpe T.W., Rathod V.K., Extraction of glycyrrhizic acid from licorice root using ultrasound: Process intensification studies. Chem. Eng. Process. Process Intensif., 2012, 54, 37-41.

[12] Dong B., Yuan X., Zhao Q., Feng Q., Liu B., Guo Y., et al., Ultrasound-assisted aqueous two-phase extraction of phenylethanoid glycosides from Cistanche deserticola Y. C. Ma stems: Sample Preparation. J. Sep. Sci., 2015, 38, 1194-1203.

[13] Sun C., Xie Y., Tian Q., Liu H., Cloud point extraction of glycyrrhizic acid from licorice root. Sep. Sci. Technol., 2007, 42, 3259-3270.

[14] Wang Q.E., Ma S., Fu B., Lee F.S.C., Wang X., Development of multi-stage countercurrent extraction technology for the extraction of glycyrrhizic acid (GA) from licorice (Glycyrrhiza uralensis Fisch). Biochem. Eng. J., 2004, 21, 285-292.

[15] Merchuk J.C., Andrews B.A., Asenjo J.A., Aqueous two-phase systems for protein separation: studies on phase inversion. J. Chromatogr. B. Biomed. Sci. App., 1998, 711, 285-293.

[16] Ruiz-Ruiz F., Benavides J., Rito-Palomares M. Scaling-up of a B-phycoerythrin production and purification bioprocess involving aqueous two-phase systems: Practical experiences. Process Biochem., 2013, 48, 738-745.

[17] Luechau F., Selective partition of plasmid DNA and RNA from crude Escherichia coli cell lysate by aqueous two-phase systems. Biochem. Eng. J., 2011, 55, 230-232.

[18] Zeng C.X., Xin R.P., Qi S.J., Yang B., Wang Y.H., Aqueous two-phase system based on natural quaternary ammonium compounds for the extraction of proteins: Other Techniques. J. Sep. Sci., 2016, 39, 648-654.

[19] Li N., Wang Y., Xu K., Huang Y., Wen Q., Ding X., Development of green betaine-based deep eutectic solvent aqueous two-phase system for the extraction of protein. Talanta, 2016, 152, 23-32.
[20] Huo Q., Lin Q., Zhao Y.E., Separation of glycyrrhizic acid by aqueous two-phase extraction with EtOH- $\mathrm{K}_{2} \mathrm{HPO}_{4}$. Fine Chem., 2002, 19, 65-61.

[21] Xie T., Wang W., Ruchun W.U., Mei L.I., Liao A., Extraction of glycyrrhizic acid using PEG-( $\left(\mathrm{NH}_{4}\right)_{2} \mathrm{SO}_{4}$ aqueous two-phase system. Chem. Res. Appl., 2005, 268-273.

[22] Sun C., Liu W.J., Zhang B., Nonionic surfactant aqueous twophase extraction of glycyrrhizic acid. Food Sci. Technol., 2014, 39, 237-241.

[23] Malpiedi L.P., Fernández C., Picó G., Nerli B., Liquid-liquid equilibrium phase diagrams of polyethyleneglycol + sodium tartrate + water two-phase systems. J. Chem. Eng. Data, 2008, 53, 1175-1178.

[24] Regupathi I., Srikanth C.K., Sindhu N., Liquid-liquid equilibrium of poly(ethylene glycol) 2000 + diammonium hydrogen citrate + water system at different temperatures. J. Chem. Eng. Data, 2011, 56, 3643-3650.

[25] Silvério S.C., Wegrzyn A., Lladosa E., Rodríguez O., Macedo E.A., Effect of aqueous two-phase system constituents in different poly(ethylene glycol)-salt phase diagrams. J. Chem. Eng. Data, 2012, 57, 1203-1208.

[26] Raja S., Murty V.R., Liquid-liquid equilibrium of poly(ethylene glycol) 6000 + sodium succinate + water System at different temperatures. Sci. World J., 2013, 2013, 1-7.

[27] Wysoczanska K., Macedo E.A., Effect of molecular weight of polyethylene glycol on the partitioning of DNP-amino acids: PEG $(4000,6000)$ with sodium citrate at 298.15 K. Fluid Phase Equilibr., 2016, 428, 84-91.

[28] Wysoczanska K., Macedo E.A., Influence of the molecular weight of PEG on the polymer/salt phase diagrams of aqueous two-phase systems. J. Chem. Eng. Data, 2016, 61, 4229-4235.

[29] Zafarani-Moattar M.T., Hamzehzadeh S., Hosseinzadeh S., Phase diagrams for liquid-liquid equilibrium of ternary poly(ethylene glycol)+di-sodium tartrate aqueous system and vapor-liquid equilibrium of constituting binary aqueous systems at $\mathrm{T}=(298.15,308.15$, and 318.15)K. Fluid Phase Equilibr., 2008, 268, 142-152.

[30] Graber T.A., Taboada M.E., Asenjo J.A., Andrews B.A., Influence of molecular weight of the polymer on the liquid-liquid equilibrium of the poly(ethylene glycol) $+\mathrm{NaNO}_{3}+\mathrm{H}_{2} \mathrm{O}$ System at 298.15 K. J. Chem. Eng. Data, 2001, 46, 765-768. 